\title{
THE SEMIGROUP OF NONEMPTY FINITE SUBSETS OF RATIONALS
}

\author{
REUBEN SPAKE
}

\author{
Department of Mathematics \\ University of California \\ Davis, California 95616
}

(Received December 8, 1986)

ABSTRACT. Let $Q$ be the additive group of rational numbers and let $\mathscr{R}$ be the additive semigroup of all nonempty finite subsets of $Q$. For $X \in \Re$, define $A_{X}$ to be the basis of $\langle X-\min (X)\rangle$ and $B_{X}$ the basis of $\langle\max (X)-X\rangle$. In the greatest semilattice decomposition of $\mathscr{R}$, let $A(\mathrm{X})$ denote the archimedean component containing $X$. In this paper we examine the structure of $\mathscr{R}$ and determine its greatest semilattice decomposition. In particular, we show that for $X, Y \in R^{R}$, $s(\mathrm{X})=A(\mathrm{Y})$ if and only if $A_{\mathrm{X}}=\mathrm{A}_{\mathrm{Y}}$ and $\mathrm{B}_{\mathrm{X}}=\mathrm{B}_{\mathrm{Y}}$. Furthermore, if $\mathrm{X}$ is a non-singleton, then the idempotent-free $A(X)$ is isomorphic to the direct product of a power joined subsemigroup and the group $Q$.

KEY WORDS AND PHRASES. Greatest semilattice decomposition, archimedean components. 1950 AMS SUBJECT CLASSIFICATION OODES. 20M14, 10L10, 05A99.

1. INTRODUCTION.

In [1] we determined the structure of the semigroup of nonempty finite subsets of integers. In this paper we extend the results of [1] for the semigroup of nonempty finite subsets of rationals. In particular, we give a complete description of its greatest semilattice decomposition. We also propose an isomorphism problem. It is assumed the reader is familiar with the basic notions on commutative semigroups and greatest semilattice decompositions; otherwise refer to $\mathrm{Clifford}$ and Preston [2] and Petrich [3]. We begin with some notation and several definitions.

Let $Q$ be the group of rational numbers, $Z$ the group of integers, and define $\Re$ to be the semigroup consisting of all nonempty finite subsets of $Q$ with the operation

$$
\mathrm{A}+\mathrm{B}=\{\mathrm{a}+\mathrm{b}: \mathrm{a} \in \mathrm{A}, \mathrm{b} \in \mathrm{B}\}, \mathrm{A}, \mathrm{B} \in \mathscr{R} .
$$

A singleton element of $\mathscr{R}$ will be identified with the rational number it contains. The semigroup $\mathscr{R}$ is a commutative countable semigroup with identity element 0 .

Let $\mathrm{X}=\left\{\mathrm{a}_{1} / \mathrm{b}_{1}, \mathrm{a}_{2} / \mathrm{b}_{2}, \ldots, \mathrm{a}_{\mathrm{n}} / \mathrm{b}_{\mathrm{n}}\right\} \in \mathscr{R}$, where $\mathrm{a}_{1} / \mathrm{b}_{1}<\cdots<\mathrm{a}_{n} / \mathrm{b}_{\mathrm{n}}$ and each $a_{i}$, $b_{i}$ are relatively prime integers, (if $X$ contains an integer $x$, then express $x$ as $x / 1)$. Define $\min (X)=a_{1} / b_{1}, \max (X)=a_{n} / b_{n}$, and let $\ell(X)$ denote the least 
(positive) common multiple of the integers $b_{1}, b_{2}, \ldots b_{n}$. If $x$ consists only of integers, then define $\operatorname{gcd}(X)$ to be the grealest (non-negative) common divisor of the integers in $X$, where $\operatorname{gcd}(0)=0$ and $\operatorname{gcil}(X \cup\{0\})=\operatorname{gcd}(X)$. Let $Z_{+}$be the set of positive integers and define the integer interval $[a, b]=\{x \in Z: a \leq x \leq b\}$ if $a, b \in Z$ with $a \leq b$. For $U \in \mathscr{R}$, let $\langle U\rangle$ denote the semigroup generated by the set $U$, and for $m \in Z_{+}, r \in Q$ define

$$
\mathrm{mU}=\underbrace{\mathrm{U}+\cdots+\mathrm{U}}_{m}, \mathrm{r} * \mathrm{U}=\{\mathrm{ru}: \mathrm{u} \in \mathrm{U}\}, \text { and } \mathrm{Z}_{\mathrm{m}}=\mathrm{Z} /\langle-\mathrm{m}, \mathrm{m}\rangle .
$$

In the greatest semilattice decomposition of $\Re$, let $A(X)$ denote the archimedean component containing $X$. Define the partial order $\leq$ on the (lower) semilattice as: $A(X) \leq A(Y)$ if and only if $\mathrm{nX}=\mathrm{Y}+\mathrm{W}$ for some $W \in \mathscr{R}$ and $\mathrm{n} \in$ $Z_{+}$(equivalently: $U+V \in A(X)$ for some (all) $U \in A(X)$ and $V \in \mathscr{A}(Y)$ ). Note that A(0) corisists of all the singletons in $\mathscr{R}$ and $A(0) \cong Q$. Moreover, since 0 is clearly the only idempotent in $\Re$, evidently $d(X)$ is idempotent-free if and only if $X$ is a non-singleton. We will show later in Theorem 2.1 that there are in fact inf initely many archimedean components in the greatest semilattice decomposition of $\Re$.

For $X \in \mathscr{R}$, define $A_{X}$ to be the basis of $\langle X-\min (X)\rangle$ and $B_{X}$ the basis of $\langle\max (X)-X\rangle$. Also, if $X$ is a non-singleton define $\operatorname{id}(X)=\min \left(A_{X} \backslash\{0\}\right)$ and $f d(X)=\min \left(B_{X} \backslash\{0\}\right)$. Note that $\ell\left(A_{X}\right)=\ell(X-x)=\ell\left(B_{X}\right)$ for all $x \in X$. When $X$ is a non-singleton, $A_{X}$ and $B_{X}$ have at most $1+\ell\left(A_{X}\right)$ id( $\left.X\right)$ and $1+\ell\left(B_{X}\right) f d(X)$ elements, respectively (if $X$ is a singleton then $A_{X}=B_{X}=\{0\}$ ). We close this introduction with an example. Let $X=\{-3 / 10,-1 / 5,4 / 5,11 / 6,2\}$. We wish to determine $A_{X}$ and $B_{X}$. First, $\ell(X)=30$, so $X=1 / 30 *\{-9,-6,24,55,60\}$. Thus

$$
X-\min (X)=1 / 30 *\{0,3,33,64,69\} \text { and }
$$$$
\max (X)-X=1 / 30 *\{0,5,36,66,69\} \text {. }
$$

Consequently, $A_{X}=1 / 30 *\{0,3,64\}=\{0,1 / 10,32 / 15\}$ and $B_{X}=1 / 30 *\{0,5,36,69\}$ $=\{0,1 / 6,6 / 5,23 / 10\}$.

\section{STRUCTURE OF $\%$.}

In this section we examine the structure of $\mathscr{R}$ by determining its greatest semilattice decomposition and describing the structure of its archimedean components. The first result gives a necessary and sufficient condition for two elements of $\mathscr{R}$ to be in the same archimedean component.

THEOREM 2.1. For $\mathrm{X}, \mathrm{Y} \in \mathscr{R}, \mathscr{A}(\mathrm{X})=\mathscr{A}(\mathrm{Y})$ if $\underline{\text { and }}$ only $\underline{\text { if }} \mathrm{A}_{\mathrm{X}}=\mathrm{A}_{\mathrm{Y}}$ and $\mathrm{B}_{\mathrm{X}}=\mathrm{B}_{\mathrm{Y}}$.

PROOF. Let $X, Y \in \mathscr{R}$ and without loss of generality assume $\min (X)=\min (Y)=0$. Let $U$ and $V$ be such that $U=\ell(X) * X$ and $V=\ell(Y) * Y$. Note that $U$ and $V$ are finite sets of integers. Suppose $A_{X}=A_{Y}$ and $B_{X}=B_{Y}$. Since $\min (X)=\min (Y)=$ 0 , this implies $\ell(X)=\ell\left(A_{X}\right)=\ell\left(A_{Y}\right)=\ell(Y)$. Hence $A_{U}=A_{V}$ and $B_{U}=B_{V}$. By [1], $A(\mathrm{U})=A(\mathrm{~V})$ and therefore it follows that $A(\mathrm{X})=A(\mathrm{Y})$.

Conversely, suppose $A(X)=\mathscr{A}(\mathrm{Y})$. There exist $\mathrm{n}, \mathrm{m} \in \mathrm{Z}_{+}$and $\mathrm{S}, \mathrm{T} \in \mathscr{R}$ such 
that

$$
\mathrm{nX}=\mathrm{Y}+\mathrm{S} \text { and } \mathrm{mY}=\mathrm{X}+\mathrm{T} .
$$

Since necessarily $\min (S)=\min (T)=0$, evidently

$$
A_{Y} \subseteq Y \subseteq Y+S \subseteq\left\langle A_{X}\right\rangle
$$

and likewise $A_{X} \subseteq\left\langle A_{Y}\right\rangle$. Consequently, $\left\langle A_{X}\right\rangle=\left\langle A_{Y}\right\rangle$ and by definition this implies $A_{X}=A_{Y}$. Similarly $B_{X}=B_{Y}$ and this completes the proof.

Using the above theorem we can determine when two archimedean components are related with respect to the order on the semilattice.

THEOREM 2.2. The following are equivalent.

(i) $\quad d(\mathrm{X}) \leq A(\mathrm{Y})$.

(ii) $\mathrm{A}_{\mathrm{Y}} \subseteq\left\langle\mathrm{A}_{\mathrm{X}}\right\rangle$ and $\mathrm{B}_{\mathrm{Y}} \subseteq\left\langle\mathrm{B}_{\mathrm{X}}\right\rangle$.

(iii) $A_{X+Y}=A_{X}$ and $B_{X+Y}=B_{X}$.

PROOF. Suppose $A(X) \leq A(Y)$. There exist $U \in \mathscr{R}$ and $\mathrm{n} \in \mathrm{Z}_{+}$such that

$$
\mathrm{n}(\mathrm{X}-\min (\mathrm{X}))=\mathrm{Y}-\min (\mathrm{Y})+\mathrm{U} .
$$

Since $\min (U)=0$,

$$
A_{Y} \subseteq Y-\min (Y) \subseteq Y-\min (Y)+U \subseteq\left\langle A_{X}\right\rangle .
$$

Similarly $B_{Y} \subseteq\left\langle B_{X}\right\rangle$. Suppose next that assertion (ii) holds. Then

$$
\mathrm{Y}-\min (\mathrm{Y}) \subseteq\left\langle\mathrm{A}_{\mathrm{Y}}\right\rangle \subseteq\left\langle\mathrm{A}_{\mathrm{X}}\right\rangle
$$

and thus

$$
A_{X} \subseteq X+Y-\min (X+Y) \subseteq\left\langle A_{X}\right\rangle .
$$

Hence $A_{X+Y}=A_{X}$. Likewise $B_{X+Y}=B_{X}$. Finally, if (iii) holds, then by Theorem 2.1 $\mathrm{X}+\mathrm{Y} \in \mathcal{A}(\mathrm{X})$; that is, $\mathscr{A}(\mathrm{X}) \leq A(Y)$ and the proof is complete.

Since $A_{Y}$ and $B_{Y}$ are finite sets, it is relatively easy to determine when $A(\mathrm{X}) \leq A(\mathrm{Y})$ using Theorem 2.2(ii). For example, let $W=\{-10 / 7,-8 / 7,22 / 7,33 / 7$, $5\}, X=\{1 / 7,5 / 21,29 / 21,68 / 21,23 / 7\}$, and $Y=\{-15,-13,8,28,30\}$. Then $\ell(W)$ $=7, \ell(X)=21$, and $\ell(Y)=1$. Thus

$$
\begin{aligned}
& W-\min (W)=1 / 7 *\{0,2,32,43,45\}, \max (W)-W=1 / 7 *\{0,2,13,43,45\}, \\
& X-\min (X)=1 / 21 *\{0,2,26,65,66\}, \max (X)-X=1 / 21 *\{0,1,40,64,66\} . \\
& Y-\min (Y)=\{0,2,23,43,45\}, \text { and } \max (Y)-Y=\{0,2,22,43,45\} .
\end{aligned}
$$

Hence, $A_{W}=\{0,2 / 7,43 / 7\}, A_{X}=\{0,2 / 21,65 / 21\}, A_{Y}=\{0,2,23\}, B_{W}=\{0,2 / 7,13 / 7\}$,

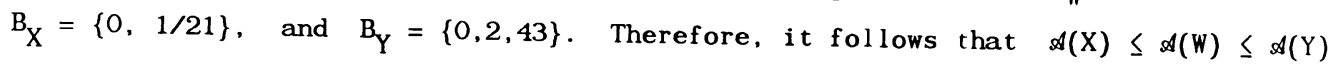
with $\mathrm{A}(\mathrm{X}) \neq A(\mathrm{~W})$ and $A(W) \neq A(\mathrm{Y})$.

Next, for $\mathrm{X} \in \mathscr{R}$ define $\mathscr{A}_{0}(\mathrm{X})=\{\mathrm{Y} \in \mathscr{A}(\mathrm{X}): \min (\mathrm{Y})=0\}$. It is clear that $A_{0}(X)$ is a subsemigroup of $A(X)$. In general, elements of $A(X)$ can be uniquely expressed in the form $U+q$, where $U \in A_{O}(X)$ and $q \in Q$. Hence it follows that $A(X) \cong A_{0}(X) \oplus Q$. Moreover, we have the following 
THEOREM 2.3. The idempotent-free archimedean component $A(X)$, where $X$ is a non-singleton, is isomorphic to the direct product of the idempotent-free power joined subsemigroup $A_{0}(\mathrm{X})$ and the group $\mathrm{Q}$.

PROOF. Let $X$ be a non-singleton with $\min (X)=0$ and let $q$ be a non-zero rational number. We will first show that $A_{0}(\mathrm{X}) \cong A_{0}(\mathrm{q} * \mathrm{X})$ under the isomorphism which maps $U$ to $q * U$. First, if $U \in A_{0}(X)$, then there exist $U_{1}, X_{1} \in \Re$ and $n$, $m \in Z_{+}$such that

$$
\mathrm{nX}=\mathrm{U}+\mathrm{U}_{1} \text { and } \mathrm{mU}=\mathrm{X}+\mathrm{X}_{1}
$$

Hence

$$
\begin{aligned}
& \mathrm{n}(\mathrm{q} * \mathrm{X})=\mathrm{q} * \mathrm{U}+\mathrm{q} * \mathrm{U}_{1} \text { and } \\
& \mathrm{m}(\mathrm{q} * \mathrm{U})=\mathrm{q} * \mathrm{X}+\mathrm{q} * \mathrm{X}_{1}
\end{aligned}
$$

giving $q * U \in A_{0}(q * X)$. It suffices therefore to show that for each $v \in A_{0}(q * X)$ there exists $V_{1} \in A_{0}(X)$ such that $V=q * V_{1}$. Let $V \in A_{0}(q * X)$. Then there exist $S, T \in \mathscr{R}$ and $s, t \in Z_{+}$such that

$$
\mathrm{q}^{*}(\mathrm{sX})=\mathrm{V}+\mathrm{S} \text { and } \mathrm{tV}=\mathrm{q} * \mathrm{X}+\mathrm{T} \text {. }
$$

Let $V_{1}, S_{1}$, and $T_{1}$ be such that $V=q * V_{1}, S=q * S_{1}$, and $T=q * T T_{1}$. Then

$$
\mathrm{sX}=\mathrm{V}_{1}+\mathrm{s}_{1} \text { and } \mathrm{tV}_{1}=\mathrm{X}+\mathrm{T}_{1} \text {. }
$$

Hence $v_{1} \in A_{0}(X)$ and consequently $A_{0}(X) \cong A_{0}(q * X)$ for each non-zero rational $q$. In particular, $A_{0}(X) \cong A_{0}(\ell(X) * X)$. Since $\ell(X) * X$ is a set of integers, by [1] $A_{0}(\ell(X) * X)$ is power joined. Therefore $A_{0}(X)$ is power joined and this completes the proof.

COROLLARY 2.4. For $\mathrm{X} \in \mathscr{R}, d(\mathrm{X}) \cong A(\mathrm{q} * \mathrm{X})$ for each non-zero rational number $\mathrm{q}$.

The following equivalence relation on $\Re$ is called the $g$-relation on $\mathscr{R}$ (sce [2] and [3] for more on the g-relation):

$$
\begin{gathered}
X \& Y \text { if and only if } X=Y+U \text { and } Y=X+V \\
\text { for some } U, V \in \Re .
\end{gathered}
$$

However, observe that if $X=Y+U$ and $Y=X+V$, then $X$ and $Y$ must necessarily be of the same cardinality since $X, Y \in \mathscr{R}$; that is, evidently $U$ and $V$ are singletons. Hence

$$
X g Y \text { if and only if } \mathrm{X}=\mathrm{Y}+\mathrm{q} \text { for some } \mathrm{q} \in \mathrm{Q} \text {. }
$$

Therefore, in $\mathscr{R}$ the $g$-class of $X$ is the set of all rational translates of $X$ (i.e. elements of the form $X+q, q \in Q$ ).

Let $\rho_{0}$ denote the least semilattice congruence on $\mathscr{R}$. Define an equivalence relation $\pi$ on $\mathscr{R}$ by

$$
X \pi Y \text { if and only if } n X=m Y \text { for some } n, m \in Z_{+} \text {. }
$$

Using Theorem 2.3 we immediately have 
THEOREM 2.5. The least semilattice congruence on $\Re \underline{\text { is }} \rho_{0}=g \pi g$. That $\underline{\text { is }}$,

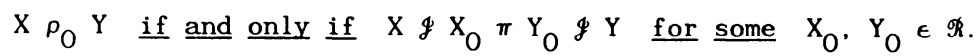

Next we look more deeply into the structure of $A(\mathrm{X})$. The structure of $A(0)$ is clear since $A(0) \cong Q$. Using the above results, evidently $Y \in A(X)$ if and only if $\mathrm{Y}-\min (\mathrm{Y})=A_{X} \cup \mathrm{Y}_{1}$ where $\mathrm{Y}_{1} \subseteq\left\langle A_{\mathrm{X}}\right\rangle$ and $\max (\mathrm{Y})-\mathrm{Y}=\mathrm{B}_{\mathrm{X}} \cup \mathrm{Y}_{2}$ where $\mathrm{Y}_{2} \subseteq\left\langle\mathrm{B}_{\mathrm{X}}\right\rangle$. More precisely we have the following direct consequence of Theorem 3.2 from [1].

THEOREM 2.6. Let $X$ be a non-singleton and $U$ be such that $X-m i n(X)=g * U$, where $g=\operatorname{gcd}\left(\ell\left(A_{X}\right) * A_{X}\right) / \ell\left(A_{X}\right)$. Define $A_{i}=\left\{x \in\left\langle A_{U}\right\rangle: x \equiv i(\bmod a)\right\}$ and $B B_{j}=\{x$ $\left.\epsilon\left\langle B_{U}\right\rangle: x \equiv j(\bmod b)\right\} \quad \underline{\text { for }} i \epsilon[0, a-1], j \in[0, b-1]$, where $a=i d(U)$ and $b=$ $\mathrm{fd}(\mathrm{U}) . \underline{\text { Let }} \mathbf{c}=\max \left\{\min \left(\mathrm{A}_{\mathbf{i}}\right): \mathbf{i} \in[0, \mathrm{a}-1]\right\} \quad \underline{\text { and }} \mathbf{d}=\max \left\{\min \left(\mathrm{B}_{\mathbf{i}}\right): \mathrm{i} \in[0, \mathrm{~b}-1]\right\}$. Then $\mathrm{Y} \in \mathscr{A}(\mathrm{X})$ if and only if there exist $\mathrm{V} \in \mathscr{K}$ and $\mathrm{n}_{0} \in \mathrm{Z}_{+}$such that $\mathrm{Y}-\mathrm{min}(\mathrm{Y})$ $=g * V$ and for all integers $n \geq n_{0}$

$$
\begin{gathered}
n V=\underset{i=0}{u}\left\{x \in A_{i}: x<c-a\right\} U[c-a+1, n \max (V)+b-d-1] \\
\quad \underset{i=0}{U_{i=1}^{U}}\left\{n \max (V)-x: x \in B_{i}, x<d-b\right\} \\
=\left\langle A_{U}\right\rangle \cap\left(\max (V)-\left\langle B_{U}\right\rangle\right) .
\end{gathered}
$$

Next we reproduce several definitions and facts from Tamura [4] that we will use in the next theorem. Let $T$ be an additively denoted idempotent-free commutative archimedean semigroup. For fixed $b \in T$, define a congruence $\rho_{b}$ on $T$ by

$$
x \rho_{b} y \text { if and only if } n b+x=m b+y \text { for some } n, m \in Z_{+} \text {. }
$$

Then $T / \rho_{b}=G_{b}$ is a group called the structure group of $T$ determined by the standard element b. Also, define a compatible partial order $\underset{b}{<}$ on $T$ by

$$
x<y \text { if and only if } x=n b+y \text { for some } n \in Z_{+} \text {. }
$$

Then $T=\underset{\lambda \in G_{b}}{U} T_{\lambda}$. equivalently $T / \rho_{b}=\left\{T_{\lambda}\right\}, \lambda \in G_{b}$, where each $T_{\lambda}$ is a discrete tree without smallest element with respect to $<$, (a discrete tree, with respect to $<$, is a lower semilattice such that for any $\underset{b}{c}<d$ the set $\{x: c \underset{b}{<} \underset{b}{<} \underset{b}{<}$ is a finite chain). Finally, define a relation $\eta$ on $T$ as follows:

$x \eta y$ if and only if $n b+x=n b+y$ for some $n \in Z_{+}$.

The relation $\eta$ is the least cancellative congruence on $T$. Let $Q_{+}$denote the set of positive rational numbers.

THEOREM 2.7. Let $\quad \underline{A} \epsilon \mathscr{R}$ be $\underline{\text { a }} \underline{\text { non-singleton }} \underline{\text { with }} \min (\mathrm{A})=0$ and $\mathrm{g}=\operatorname{gcd}(\ell(\mathrm{A}) * \mathrm{~A}) / \ell(\mathrm{A})$. The structure group of $A_{0}(\mathrm{~A})$ determined by the standard 


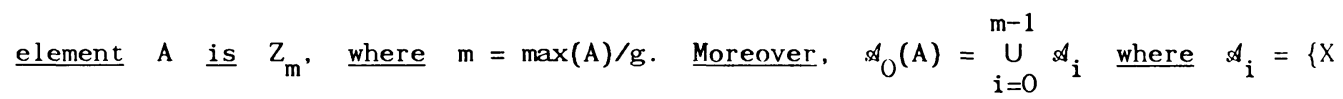
$\left.\epsilon A_{0}(\mathrm{~A}): \max (\mathrm{X}) / \mathrm{g} \equiv \mathrm{i}(\bmod \mathrm{m})\right\} \quad \underline{\text { is }}$ a discrete tree without $\underline{\text { smallest }}$ element $\underline{\text { with }}$ respect to $<$. Furthermore, the structure group of $A(A)$ determined by the $\underline{\text { standard element }} \quad A$ is $Q \oplus Z_{m}$.

PROOF. This follows from $[1]$ since $\mathscr{A}_{0}(\mathrm{~A}) \cong \mathscr{A}_{0}(1 / \mathrm{g} * \mathrm{~A})$.

Using Theorem 2.7 we have the immediate

PROPOSITION 2.8. Let $A$ be a non-singleton. The homomorphism $\mathrm{h}: A_{0}(\mathrm{~A}) \rightarrow_{+}$ defined by $h(X)=\max (X)$ is the greatest cancellative homomorphism. That $\underline{\text { is }}$. the relation $\eta$ on $A_{0}(\mathrm{~A})$ defined by

$X \eta Y$ if and only if $\max (\mathrm{X})=\max (\mathrm{Y})$

is the least cancellative congruence. Moreover, the relation $\sigma$ on $A(A)$ defined by

$$
\begin{gathered}
X \sigma Y \text { if and only } \underline{\text { if }} \min (X)=\min (Y) \text { and } \\
\max (X)=\max (Y)
\end{gathered}
$$

$\underline{\text { is }}$ the least cancellative congruence. The semigroups $A_{0}(\mathrm{~A}) / \eta$ and $A(\mathrm{~A}) / \sigma$ are $\underline{\text { idempotent }}-\underline{\text { free }}$ commutative archimedean cancellative semigroups.

For a description of the greatest cancellative homomorphic image of $\mathscr{A}_{0}(\mathrm{~A})$ we direct the reader to [1]. We close this report with an open isomorphism problem. Any partial solutions would be appreciated.

PROBLEM. For X,Y $\in \mathscr{R}$, under what conditions will $\mathscr{A}(\mathrm{X})$ be isomorphic to $\mathscr{A}(\mathrm{Y})$ ? See Theorem 5.5 of [5] for some related results and also recall Corollary 2.4.

\section{REFERENCES}

1. SPAKE, R. The Semigroup of Nonempty Finite Subsets of Integers, Internat. I. Math. and Math. Sci. $\underline{9}$ (1986), 605-616.

2. CLIFFORD, A.H. and PRESTON, G.B. The Algebraic Theory of Semigroups, Amer. Math. Soc., 1961.

3. PETRICH, M. Introduction to Semigroups, Merrill, 1973.

4. TAMURA, T. Construction of Trees and Commutative Archimedean Semigroups, Math. Nachr. 36 (1968), 255-287.

5. SPAKE, R. Idempotent-free Archimedean Components of the Power Semigroup of the Group of Integers 1, Math. Japon. 31 (1986), 791-810. 


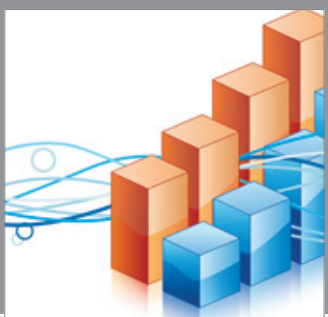

Advances in

Operations Research

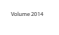

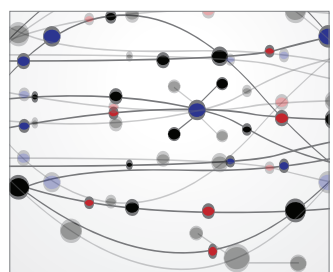

\section{The Scientific} World Journal
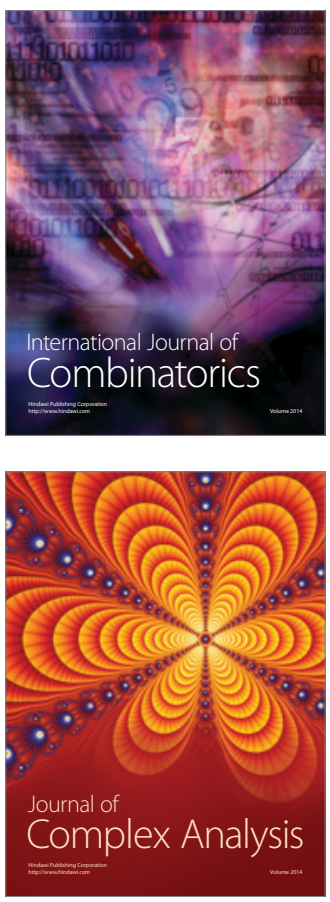

International Journal of

Mathematics and

Mathematical

Sciences
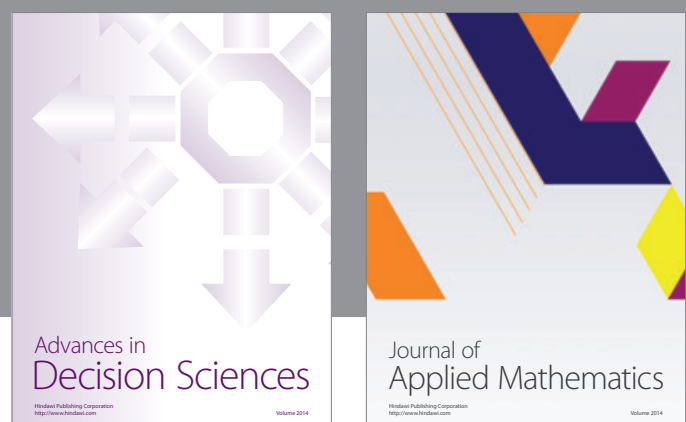

Journal of

Applied Mathematics
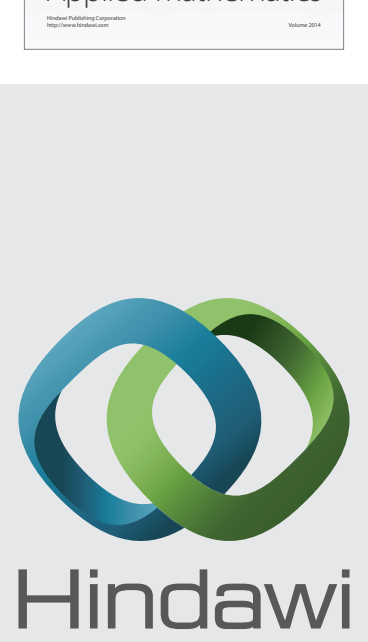

Submit your manuscripts at http://www.hindawi.com
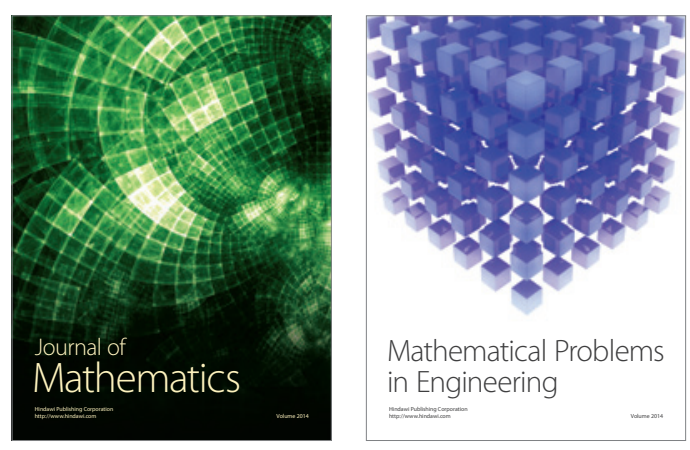

Mathematical Problems in Engineering
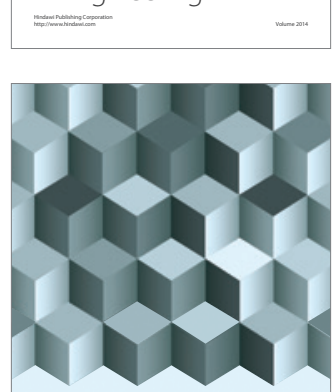

Journal of

Function Spaces
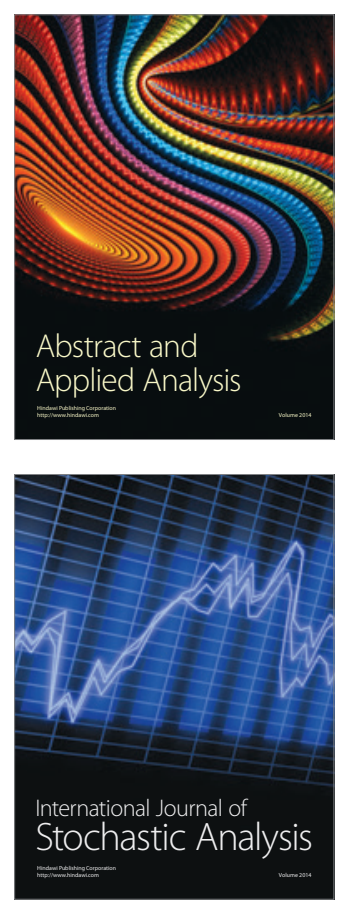

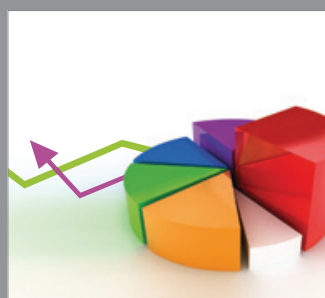

ournal of

Probability and Statistics

Promensencen
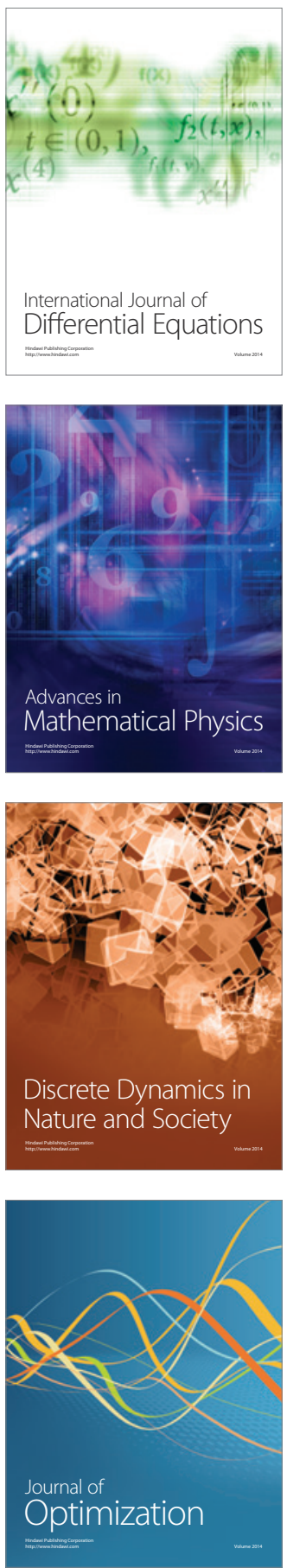